\title{
CARACTERÍSTICAS CLÍNICAS DE VULVOVAGINITIS POR CÁNDIDA ALBICANS EN MUJERES EN EDAD REPRODUCTIVA
}

\author{
Nathaly Lisseth Barraza Guimarrea ${ }^{1,3}$, Félix Ayala Peralta ${ }^{2,3, b}$, Humberto Izaguirre Lucano ${ }^{2, c}$, \\ Antonio Luna Figueroa ${ }^{2,3, b}$, César Carranza Asmat',3,b.
}

\begin{abstract}
RESUMEN
Objetivos. Determinar las características clínicas de la infección vulvovaginal por Candida albicans (CVV) en mujeres en edad reproductiva. Materiales y métodos. Investigación observacional, descriptiva, retrospectiva y transversal. Se incluyó una muestra de 120 mujeres con diagnóstico de infección vulvovaginal por Candida Albicans atendidas en consulta externa en el Instituto Nacional Materno Perinatal periodo 2018. Resultados. La prevalencia de infección vulvovaginal por Candida albicans fue $28 \%$. Las características sociodemográficas fueron, edad mediana con $53,3 \%$, estado civil conviviente $39,2 \%$, instrucción superior $49,2 \%$ y ama de casa $53,3 \%$. Respecto a las características ginecológicas incluyeron número de parejas sexuales $2,1 \pm 0,8$, uso de anticonceptivo depoprovera con $35 \%$ y anticonceptivo oral combinado $28,3 \%$; hábitos de higiene íntima ocasional $61,7 \%$. La sintomatología predominante fue prurito en $100 \%$, flujo genital blanquecino $99,2 \%$ e irritación vulvar $55,7 \%$. Conclusiones. La prevalencia de CVV fue $28 \%$. Sintomatología clínica predominante fue prurito vulvar y flujo genital balnquecino.
\end{abstract}

Palabras clave: Prevalencia; Candidiasis; vulvovaginal; Candida Albicans (Fuente: DeCS BIREME).

\section{CLINICAL CHARACTERISTICS OF VULVOVAGINITIS DUE TO CANDIDA ALBICANS IN WOMEN OF REPRODUCTIVE AGE}

\begin{abstract}
Objectives. To determine the clinical characteristics of vulvovaginal infection by Candida albicans (CVV) in women of reproductive age. Materials and methods. Observational, descriptive, retrospective and transversal research. A sample of 120 women diagnosed with vulvovaginal infection by Candida Albicans who attended the outpatient clinic at the National Maternal and Perinatal Institute period 2018 was included. Results. The prevalence of vulvovaginal infection by Candida albicans was $28 \%$. The sociodemographic characteristics were, median age with $53.3 \%$, cohabiting civil status $39.2 \%$, higher education $49.2 \%$ and housewife $53.3 \%$. Regarding the gynecological characteristics, they included the number of sexual partners $2.1 \pm 0.8$, the use of depoprotein contraceptive with $35 \%$ and combined oral contraceptive $28.3 \% ; 61.7 \%$ casual intimate hygiene habits. The predominant symptomatology was itching in 100\%, whitish genital discharge $99.2 \%$ and vulvar irritation $55.7 \%$. Conclusions. The prevalence of CVV was $28 \%$. The predominant clinical symptomatology was vulvar pruritus and balnquecino genital flow.
\end{abstract}

Keywords: Prevalence; Candidiasis; vulvovaginal; Candida Albicans (Source: MeSH NLM).

\section{INTRODUCCIÓN}

En la actualidad, a pesar de los grandes avances en la tecnología del diagnóstico de la evidencia micológica, la candidiasis vulvovaginal (CVV) en la mujer constituye un problema de alta frecuencia en salud pública mundial y nacional, porque está relacionada directamente con mujeres en edad fértil (MEF) y sexualmente activas; cuyas infecciones a repetición pueden causar alteraciones en la superficie de las células de la mucosa cérvico-vaginal o inflamación, y que éstas a largo plazo, podrían contribuir a la presencia de lesiones premalignas en el cuello uterino de la mujer ${ }^{1,2}$
Identificar la prevalencia de la CVV nos permitirá estudiar la población más afectada de las mujeres y así evitar futuras complicaciones en la mujer, por ejemplo, una infección vulvovaginal si no es prevenida o diagnosticada para el tratamiento adecuado puede llevar a una enfermedad inflamatoria pélvica u otra enfermedad, ocasionando problemas de fertilidad en la mujer ${ }^{3,4}$.

A pesar de una lista creciente de casos sobre VVC, existen pocos estudios en nuestro medio relacionados a esta patología que continúa afectando millones de MEF. En tal sentido la presente investigación servirá para identificar la prevalencia de la infección vulvovaginal por Candida

Universidad Privada San Juan Bautista. Lima- Perú.

Universidad Nacional Mayor de San Marcos. Lima-Perú

Instituto Nacional Materno Perinatal. Lima-Perú.

a Médico Cirujano. Ex interno de Medicina del Instituto Nacional Materno Perinatal. Lima-Perú.

b Médico GínecoObstetra. Profesor ordinario del Departamento Académico de Ginecología y Obstetricia de la Facultad de Medicina. Universidad Nacional Mayor de San Marcos. Lima-Perú.

Médico Gíneco Obstetra. Jefe del Servicio de Ginecología Patológica del Instituto Nacional Materno Perinatal. Lima-Perú.

Citar como: Barraza NL, Ayala-Peralta F, Izaguirre H, Luna A, Carranza C. Características clínicas de vulvovaginitis por Cándida Albicans en mujeres en edad reproductiva. Rev Peru Investig Matern Perinat 2019; 8(1):8-12. DOI: https://doi.org/10.33421/inmp.2019133 
albicans en MEF atendidas en el servicio de ginecología del Instituto Nacional Materno Perinatal en el periodo 2018, con la finalidad de contribuir con datos que puedan servir de base para establecer posibles protocolos de manejo, prevención y tratamiento de la salud pública ${ }^{5,6}$.

\section{MATERIALES Y MÉTODOS}

Tipo de investigación: Observacional, descriptivo, transversal.

Población: Todas las mujeres en edad fértil con diagnóstico de vulvovaginitis atendidas en consultorio externo del Servicio de ginecología general en el Instituto Nacional Materno Perinatal en el periodo comprendido entre enero a diciembre de $2018(\mathrm{~N}=435)$.

Criterios de Inclusión: Mujeres en edad fértil (15 a 50 años), mujeres con diagnóstico clínico de candidiasis vulvovaginal, toda mujer que fue atendida en consultorio externo del Servicio de ginecología general en el Instituto Nacional Materno Perinatal en el periodo comprendido entre enero a diciembre de 2018 , historia clínica con datos completos de las variables de estudio.

Criterios de Exclusión: Gestantes, mujeres con hemorragia vulvovaginal.

Se calculó el tamaño de la muestra, con una población de 435 y con una frecuencia esperada de $75 \%$ según referencia bibliográfica, en donde se utilizó como margen de error el $5 \%$ y con un nivel de confianza $95 \%$. Se obtuvo una muestra de 120. El muestreo fue no probabilístico por conveniencia.

Técnicas e instrumentos de recolección de datos: La técnica para realizar esta investigación fue la recolección de datos de las historias clínicas de las pacientes atendidas en consultorio externo de ginecología general. El instrumento que se utilizó fue una ficha de recolección de dato, la cual fue validada por un especialista, un metodólogo y una estadística. Para poder realizar la recolección de datos se presentó el proyecto de tesis a las autoridades pertinentes del Instituto Nacional Materno Perinatal. Se presentó una solicitud al departamento de Investigación y Ética, como también al departamento de Estadística para poder adquirir las historias clínicas y de esa manera identificar a las pacientes con vulvovaginitis. Paso seguido, se contó con la aprobación del Comité de Investigación y Ética del Instituto Nacional Materno Perinatal y se utilizó una ficha de recolección de datos previamente validada para registrar esta información.

Por último, los datos recolectados fueron incorporados a la base de datos en el programa SPSS v25, para así poder estudiar las variables realizar el análisis estadístico. En el análisis descriptivo para las variables cualitativas (Candidiasis vulvovaginal, estado civil, grado de instrucción, ocupación, hábitos de higiene íntima, diabetes mellitus, portador de VIH) se estimaron la distribución por frecuencias. Para las variables cuantitativas (Edad, número de parejas sexuales, uso de anticonceptivos) se estimaron las medidas de tendencia central (media, mediana) y de dispersión (desviación estándar).

Aspectos Éticos: Los datos recolectados para el estudio fueron almacenados con acceso restringido, para proteger la confidencialidad de los participantes. Así mismo, esta investigación fue aprobada por un comité de ética y asimismo, contó con la aprobación de la máxima autoridad del establecimiento de salud.

\section{RESULTADOS}

La población del estudio estuvo conformada por 120 mujeres en edad fértil con diagnóstico de vulvovaginitis por Candida albicans atendidas en consulta externa en servicio de ginecología general del Instituto Nacional Materno Perinatal durante el periodo 2018. Se encontró que el $28 \%$ de mujeres en edad fértil con vulvovagitnis fue causada por Candida albicans.

Tabla 1. Prevalencia de vulvovaginitis por candida albicans en mujeres en edad reproductiva atendidas en consultorio externo de ginecología general.

\begin{tabular}{lcc}
\hline VULVOVAGINITIS POR CANDIDA ALBICANS & $\mathbf{n}$ & $\%$ \\
\hline No & 315 & $72 \%$ \\
Sí & 120 & $28 \%$ \\
\hline
\end{tabular}

Tabla 2. Características sociodemográficas de mujeres en edad reproductiva con CVV atendidas en ginecología general en el Instituto Nacional Materno Perinatal durante el periodo 2018.

\begin{tabular}{lcc}
\hline Características sociodemográficas & \\
\hline \multirow{2}{*}{ Edad } & \multicolumn{2}{c}{$33,6( \pm 7,3)$} \\
\cline { 2 - 3 } & $\mathbf{n}$ & $\%$ \\
\hline Rango de Edad & \\
\hline Mediana edad (20-34 años) & 64 & $53,3 \%$ \\
\hline Añosa ( $\geq 35$ años) & 56 & $46,7 \%$ \\
\hline Estado civil & & \\
$\quad$ Soltera & 47 & $39,2 \%$ \\
\hline Conviviente & 47 & $39,2 \%$ \\
\hline Casada & 26 & $21,7 \%$ \\
\hline Grado de instrucción & & \\
\hline Primaria & 4 & $3,3 \%$ \\
\hline Secundaria & 57 & $47,5 \%$ \\
\hline Superior & 59 & $49,2 \%$ \\
\hline Ocupación & & \\
\hline Ama de casa & 64 & $53,3 \%$ \\
\hline Independiente & 23 & $19,2 \%$ \\
\hline Estudiante & 5 & $4,2 \%$ \\
\hline Empleada & 28 & $23,3 \%$ \\
\hline
\end{tabular}


El promedio de edad fue de 33,6 años (DE $\pm 7,3$ ). El $53,3 \%$ de pacientes fue de mediana edad (20-34 años). El 39,2 de pacientes correspondieron a estado civil tanto soltera como conviviente respectivamente. El 49,2\% de las pacientes fue de grado de educación superior. El $53,3 \%$ de pacientes fue de ocupación ama de casa.

Tabla 3. Características ginecológicas de mujeres en edad reproductiva con CVV atendidas en ginecología general en el Instituto Nacional Materno Perinatal durante el periodo 2018

\begin{tabular}{lcc}
\hline Características ginecológicas & \multicolumn{2}{c}{$2,1 \pm 0,8$} \\
\hline \multirow{2}{*}{ Número de parejas } & $\mathbf{n}$ & $\%$ \\
\hline Uso de anticonceptivo & & \\
$\quad$ Depoprovera & 42 & $35,0 \%$ \\
AOC & 34 & $28,3 \%$ \\
Barrera & 18 & $15,0 \%$ \\
DIU & 11 & $9,2 \%$ \\
Inyectable mensual & 8 & $6,7 \%$ \\
Otro anticonceptivo & 7 & $5,8 \%$ \\
Hábitos de higiene íntima & & \\
$\quad$ Diario & 46 & $38,3 \%$ \\
$\quad$ Ocasional & 74 & $61,7 \%$ \\
\hline
\end{tabular}

AOC: Anticonceptivo oral combinado. DIU: Dispositivo intrauterino.

El promedio del número de parejas que tuvieron las pacientes atendidas fue de 2,1. Con relación al uso de métodos anticonceptivos el $35 \%$ de pacientes utilizó depoprovera, seguida de anticonceptivo oral combinado. Respecto a los hábitos de higiene íntima, el $61,7 \%$ de pacientes fueron ocasionales.

Tabla 4. Manifestaciones clínicas de mujeres en edad reproductiva con CVV atendidas en ginecología general en el Instituto Nacional Materno Perinatal durante el periodo 2018.

\begin{tabular}{lcc}
\hline Manifestaciones clínicas & $\mathbf{n}$ & $\%$ \\
\hline Prurito vulvar & 120 & $100,0 \%$ \\
\hline Flujo blanquecino & 119 & $99,2 \%$ \\
\hline Irritación & 62 & $51,7 \%$ \\
Disuria & 16 & $13,3 \%$ \\
\hline Dispareunia & 6 & $5,0 \%$ \\
\hline
\end{tabular}

Lla sintomatología clínica predominante fue prurito vulvar seguida de flujo blanquecino e irritación vulvar.

\section{DISCUSIÓN}

La candidiasis vulvovaginal (CVV) es una infección causada por especies de candida que afecta a muchas mujeres cada año y es una de las causas más comunes de vaginitis; y su incidencia ha incrementado notablemente durante las últimas tres décadas. En la patogenia del desarrollo de CVV se atribuyen a la alteración del equilibrio entre la colonización vaginal por Candida y el entorno del huésped por cambios fisiológicos o no fisiológicos. Se han propuesto varios factores de riesgo relacionados con el hospedador y de comportamiento como factores predisponentes para CVV.

Con relación a la prevalencia de CVV en mujeres atendidas en el Instituto Nacional Materno Perinatal durante el periodo 2018 se estima que el $28 \%$ de mujeres en edad reproductiva con vulvovaginitis fue causada por Candida albicans; que coincide con los reportes recientes de Gonçalves et $a^{6}{ }^{6}$, quienes señalan que la incidencia de CVV en mujeres sintomáticas varían según localización geográfica y las poblaciones estudiadas, oscilando entre el $12,1 \%$ y el $57,3 \%$. Asimismo, dichos autores reportaron que las incidencias más altas de CVV se notificaron en estudios epidemiológicos realizados en países africanos como Nigeria por Okungbowa $\mathrm{FI}$ y $\mathrm{col}^{7}$ y en Tunisia por Amouri I y $\mathrm{col}^{8}$ con prevalencia de $57,3 \%$ y $48,0 \%$, respectivamente; seguidos de Brasil por Andrioli $\mathrm{JL}^{\mathrm{y}} \mathrm{col}^{9}$ con $47,9 \%$; Rosa $\mathrm{MI}$ y col ${ }^{10}$ con $43.5 \%$. Las prevalencias más bajas se registraron en los países europeos como Grecia reportado por Grigoriou $\mathrm{O}$ y col ${ }^{11} \mathrm{e}$ Italia por Tibaldi C y col ${ }^{12}$, con $12,1 \%$ y $18,1 \%$, respectivamente e India reportado por Ahmad A y col ${ }^{13}$ el $17,7 \%$; Vijaya D y col ${ }^{14}$ el $20,4 \%$.

Entre las características ginecológicas para CVV presentes en nuestro estudio salta a la vista el número de parejas sexuales de 2,1 $\pm 0,8$; que coinciden con reportes de Moreira $\mathrm{R}$ y $\mathrm{col}^{15}$, donde múltiples parejas sexuales se asociaron a vaginosis bacteriana y candidiasis vulvovaginal.

Con respecto al uso de anticonceptivos, en nuestro estudio tuvo mayor prevalencia la depoprovera y anticonceptivos orales combinados que son de composición hormonal que coinciden con reportes de Jacob $L$ y $\operatorname{col}^{16}$ quienes encuentran que el uso de anticonceptivos orales tuvo mayor prevalencia entre las mujeres con candidiasis vulvovaginal; así como lo señalado por Huaranga $\mathrm{M}$ y col${ }^{5}$ y Herrera $Y^{17}$, que el uso de AOC es un factor de riesgo asociado a CVV. Así como lo señalado por la Ilkit M y col${ }^{4}$ que el uso de anticonceptivos orales como el factor de riesgo para el desarrollo de esta afección.

Cabe precisar lo señalado por Gonçalves B y $\mathrm{col}^{6}$, que los posibles factores de riesgo para la infección por CVV incluyen uso de anticonceptivos orales, que pueden aumentar la adhesión de candida y la formación de hifas; dispositivos intrauterinos, que pueden actuar como un reservorio para candida permitiendo la adhesión de la levadura y la formación de biofilms; espermicida/ condones, que pueden alterar el equilibrio de la microbiota vaginal y las levaduras metabolizan el espermicida para aumentar la adherencia. 
Estudios recientes publicados por Pineda $\mathrm{J}$ y col ${ }^{18}$ señalan como factores predisponentes para CVV el embarazo, inicio de actividad sexual, uso de anticonceptivos orales o locales como el dispositivo intrauterino, uso excesivo de antibióticos de amplio espectro, mujeres obesas y aquellas mujeres que padecen diabetes mellitus, inmunosupresión (VIH) y los hábitos de higiene.

Otro factor de riesgo importante son los hábitos de higiene íntima que en nuestro estudio el $61,7 \%$ realizan en forma ocasional. Dentro de ello podemos mencionar lo reportado por Gonçalves $\mathrm{B}$ y $\mathrm{col}^{6}$, que existen otros posibles factores de riesgo de comportamiento personal que también influyen como son: higiene personal pobre, uso de ropa no transpirable, hipersensibilidad local y reacción alérgica, polimorfismos en genes de inmunidad y en antígenos de grupos sanguíneos de Lewis.

Dentro de la clínica de CVV podemos mencionar que la infección por CVV sintomática aguda implica una alteración en el equilibrio normal de la colonización por candida y el microbiota vaginal ya sea por sobrecrecimiento desencadenado de candida, alteración en los mecanismos de defensa de protección del huésped debido a predisposición genética, embarazo, uso de anticonceptivos y antibióticos e inmunosupresión.

En nuestro estudio los síntomas más predominantes en mujeres con CVV fueron prurito vulvar (100\%), flujo blanquecino $(99,2 \%)$ e irritación $(51,7 \%)$; las cuales coinciden con lo reportado por Gonçalves B y Col ${ }^{6}$ donde mencionan que los síntomas más comunes incluyen: prurito vulvovaginal, secreción vaginal, Irritación y dolor vaginal, dispareunia y disuria externa; tal como lo señala Grigoriou $\mathrm{O}$ y col${ }^{19}$ siendo su principal síntoma prurito vulvar, seguido de flujo vaginal y dispareunia.

En conclusión, podemos mencionar que la prevalencia de CVV fue $28 \%$ y la sintomatología clínica predominante fue prurito vulvar y flujo genital blanquecino.

Agradecimientos: Al Dr. Enrique Guevara Ríos, Director General del Instituto Nacional Materno Perinatal por permitir desarrollar la presente investigación. Al Dr Juan Torres, por revisar el aspecto metodológico del proyecto de investigación.

\section{Financiamiento: Autofinanciado.}

Conflicto de interés: Los autores declaran no tener algún conflicto de intereses.

\section{REFERENCIAS BIBLIOGRÁFICAS}

1. Hernández B, Prieto MA, Curiel E, Muñoz J, Quesada G, Arias MD. Perfil clínico-epidemiológico y taxonómico de la candidiasis sistemática en una unidad de cuidados intensivos. Med Intensiva. 2009;33(3):144-7.
2. BermúdezLG, Caba DA, Enríquez HC, Palma RJ, Nava F. Factores asociados a candidiasis vaginal en mujeres en edad fértil en el servicio de ginecología del Centro de Salud San Jorge del Municipio de Zudáñez en el bimestre octubrenoviembre del 2012. Archivos Bolivianos de Medicina. 2012;18(86):7-16.

3. Pappas PG, Kauffman CA, Andes D, et al. Clinical practice guidelines for the management of candidiasis: 2016 update by the Infectious Diseases Society of America. Clin Infect Dis. 2016;62(4):1.

4. Ilkit $M$, Baris $A$. The epidemiology, pathogenesis, and diagnosis of vulvovaginal candidosis: A mycological perspective. Critical Reviews in Microbiology. 2011; 37 (3):250-261.

5. Huaranga M, Domingo DA. Prevalencia y distribución de los factores de riesgo de vulvovaginitis en mujeres de edad reproductiva en el servicio de ginecología y obstetricia en el Hospital José Agurto Tello año 2015. Tesis de Pregrado. Universidad Privada San juan Bautista. 2016.

6. Gonçalves B, Ferreira C, Alves CT, Henriques M, Azeredo J, Silva S. Vulvovaginal candidiasis: Epidemiology, microbiology and risk factors. Critical Reviews in Microbiology. 2016; 42 (6):905-927.

7. Okungbowa FI, Isikhuemhen OS, Dede AP. The distribution frequency of Candida species in the genitourinary tract among symptomatic individuals in Nigerian cities. Rev Iberoam Micol. 2003;20: 60-3.

8. Amouri I, Sellami H, Borji N, et al. Epidemiological survey of vulvovaginal candidosis in Sfax, Tunisia. Mycoses. 2011; 54:499-505.

9. Andrioli JL, Oliveira GSA, Barreto CS, et al. Frequenncia de leveduras em fluido vaginal de mulheres com e sem suspeita clı́nica de candidı'ase vulvovaginal. Rev Bras Ginecol e Obs. 2009;31:300-4.

10. Rosa MI da, Rumel D. Fatores associados a' candidıase vulvovaginal: estudo exploratorio. Rev Bras Ginecol Obs. 2004;26:65-70.

11. Grigoriou O, Baka S, Makrakis E, et al. Prevalence of clinical vaginal candidiasis in a university hospital and possible risk factors. Eur J Obstet Gynecol Reprod Biol. 2006;126:121-5.

12. Tibaldi C, Cappello N, Latino MA, et al. Vaginal and endocervical microorganisms in symptomatic and asymptomatic non-pregnant females: risk factors and rates of occurrence. Clin Microbiol Infect. 2009; 15:670-9.

13. Ahmad A, Khan AU. Prevalence of Candida species and potential risk factors for vulvovaginal candidiasis in Aligarh, India. Eur J Obstet Gynecol Reprod Biol. 2009;144:68-71.

14. Vijaya D, Dhanalakshmi TA, Kulkarni S. Changing trends of vulvovaginal candidiasis. J Lab Physicians. 2014; 6:28-30.

15. Moreira R, Cunha M, Borges da Costa B, Weyll R, Ferreira $\mathrm{T}$, Silva $\mathrm{F}$, et al. Prevalence and Risk Factors for Bacterial Vaginosis and Other Vulvovaginitis in a Population of Sexually Active Adolescents from Salvador, Bahia, Brazil. Infectious Diseases in Obstetrics and Gynecology Volume. 2012, Article ID 378640, 6 pages.

16. Jacob L, John M, Kalder M, Kostev K. Prevalence of vulvovaginal candidiasis in gynecological practices in Germany: A retrospective study of 954,186 patients. Curr Med Mycol. 2018; 4(1): 6-11.

17. Herrera Y. Factores asociados, perfil clínico, laboratorial y terapéutico de flujo vaginal patológico en reclusas del penal de mujeres Cusco, 2017. Tesis de pregrado. Universidad nacional de San Antonio Abad del Cusco. 2017. 
18. Pineda J, Cortés Arturo, Castañón Laura. Candidiosis vaginal. Revisión de la literatura y situación de México y otros países latinoamericanos. Rev. Méd. Risaralda. 2017; 23 (1): $38-44$.

19. Grigoriou O, Baka S, Makrakis E, et al. Prevalence of clinical vaginal candidiasis in a university hospital and possible risk factors. Eur J Obstet Gynecol Reprod Biol. 2006;126:121-5.

\section{Correspondencia:}

Dirección: Jr. Augusto Aguire N 3268- San Martín de Porres.

Lima

Correo electrónico: nathalybarraza91@gmail.com.

Teléfono: 961739563 